\title{
Ein konzeptionelles Framework zum Verständnis des multidimensionalen Gegenstandes des Wertbeitrags der IT
}

\author{
Stefan Bartsch' ${ }^{1}$ Daniel Schlagwein ${ }^{2}$ \\ ${ }^{1}$ Institut für Wirtschaftsinformatik, Philipps-Universität Marburg \\ 2Seminar für Wirtschaftsinformatik und Informationsmanagement, \\ Universität ₹u Köln
}

\section{Einleitung}

Obwohl der Wertbegriff sowohl in der Praxis als auch in vielen wissenschaftlichen Disziplinen zur Anwendung kommt, ist die eigentliche Bedeutung nicht eindeutig (Heyde 1926, S. 7). Diese Unschärfe ist in der Anwendungsdomäne der IT ebenfalls zu erkennen (Krcmar 2005, S. 395). Es zeigt sich, dass sowohl in der Theorie als auch in der Praxis unterschiedliche Annahmen über die Wirkungsweise des ITEinsatzes existieren, die unter dem Sammelbegriff Wertbeitrag der IT diskutiert werden. Der zugrunde liegende Wertbegriff bildet die Basis für das Verständnis des Wertbeitrags der IT.

Untersuchungen zum Wertbeitrag der IT stammen überwiegend aus dem angloamerikanischen Raum und werden vornehmlich unter dem Begriff IT Business Value geführt (Johannsen et al. 2007, S. 7; Pfeifer 2003, S. 17). Der Gegenstand des Begriffs, oder Teile dessen, werden im deutschsprachigen Raum auch unter den Stichworten Nutzen, Leistung, Value, Performance, Benefit, Produktivität, Effizienz und Effektivität diskutiert. Eine inhaltliche Abgrenzung erscheint dabei nicht offensichtlich. Es existiert zwar eine Fülle an Veröffentlichungen, die sich mit Methoden zur Bewertung der IT auseinandersetzen, eine klare Definition oder intensive Auseinandersetzung hinsichtlich des Gegenstandes des Wertes und des darauf basierenden Wertbeitrags liegen in wissenschaftlichen Arbeiten in der Regel jedoch nicht vor (Bannister und Remenyi 2000, S. 232; Cronk und Fitzgerald 1997, S. 408-409). Vielmehr wird die eigentliche Bedeutung als selbsterklärend oder gegeben angenommen. Gammelgård et al. (2006, S. 3) identifizieren über 625 unterschiedliche Sichtweisen des IT Wertbeitrags. Es ist zu erkennen, dass sich diese in verschiedenen Dimensionen unterscheiden. Der wissenschaftliche Erkenntnisstand zum Gegenstand des Wertebeitrags der IT kann insgesamt als gering eingestuft werden (Kleinschmidt und Pfeifer 2004, S. 1 und S. 13). 
In der Praxis spiegelt sich dieses Bild wieder: so zeigt eine aktuelle Studie vom ITGovernance Institut (ITGI), dass in weniger als der Hälfte der befragten Unternehmen ein gemeinsames Verständnis des Wertbeitrags der IT verankert ist (Kessinger et al. 2009). Tallon et al. (2000) stellen fest, dass der Beitrag der IT von verschiedenen Gruppen innerhalb einer Organisation unterschiedlich wahrgenommen wird. Diese unterschiedlichen Sichtweisen werden in der Praxis jedoch nicht expliziert, so dass kein Austausch über den Gegenstand des Wertbeitrags der IT stattfindet und es zu unterschiedlichen Erwartungen oder gar Missverständnissen kommt.

Die Unklarheit des Gegenstandes des Wertbeitrags der IT hat einen deutlichen Einfluss auf die Steuerung der IT. So zeigt die erwähnte Studie des ITGI, dass zwei Drittel der befragten Unternehmen ihren Wertbeitrag nicht vollständig steuern (Kessinger et al. 2009). Für eine wertorientierte Steuerung der IT ist es jedoch zwingend erforderlich, ein klares Verständnis des Gegenstandes zu etablieren, und die zugrunde liegenden Steuerungsobjekte zielgerichtet darauf auszurichten.

\section{Zielsetzung und Forschungsdesign}

Dieser Beitrag zielt darauf ab, den Begriff des Wertbeitrags der IT aus Unternehmenssicht ${ }^{1}$ herzuleiten, Gründe für die verschiedenen Sichtweisen aufzuzeigen, und die Dimensionen in denen sich die Sichtweisen des Wertbeitrag der IT unterscheiden zu identifizieren. Durch Konstruktion eines Frameworks sollen diese unterschiedlichen Sichtweisen des Wertbeitrags der IT integriert dargestellt werden. Das Framework soll für unterschiedliche Beteiligte innerhalb eines Unternehmens adaptierbar und leicht verständlich sein. Es soll Hilfestellung bei der unternehmensspezifischen Ausgestaltung und Kommunikation des Wertbeitrags der IT in Unternehmen geben.

Der Prozess der Framework-Entwicklung beinhaltet idealerweise eine angemessene theoretische Basis, auf der das Framework beruht. Dazu wird in diesem Beitrag zunächst ein abstrakter Wertbegriff erarbeitet, in dem grundsätzliche, den Wert betreffende Charakteristika aufgezeigt werden. Der abstrakte Wertbegriff wird dann auf den betriebswirtschaftlichen Bereich übertragen. Dieser bildet den Rahmen für die Diskussion des Wertbeitrags der IT. Im konstruktionsorientierten Teil des Beitrags wird der Begriff des Wertbeitrags der IT konkretisiert, seine unterschiedlichen Dimensionen identifiziert und die Ergebnisse in einem Framework integriert.

Aufgrund der konstruktionsorientierten Zielsetzung des Beitrags dient der Design-Science-Ansatz von Hevner et al. (2004) als Basis zur Problemlösung. Die Vorgehensweise orientiert sich an der Design Science Research Methodology for Information Systems Research (DSRM) von Peffers et al. (2007), welche die Pro-

${ }^{1}$ Eine makroökonomische Sichtweise ist nicht Gegenstand dieses Beitrags. 
zessschritte: Problemidentifikation und Motivation, Zieldefinition, Modellentwicklung, Demonstration, Evaluation und Kommunikation vorsieht. Die einzelnen Schritte sind durch die Abschnitte dieses Beitrags abgebildet. Die Schritte Demonstration und Evaluation sind im letzten Kapitel zusammengefasst dargestellt. Der letzte Punkt, die Kommunikation der Ergebnisse, wird durch die Publikation dieses Beitrags vollzogen.

\section{Entwicklung eines Frameworks zum Verständnis des Gegenstandes des Wertbeitrags der IT}

\subsection{Der abstrakte Wertbegriff}

Abstrakt lässt sich Wert als eine Sache beschreiben, die zur Bedürfnisbefriedigung oder Existenzerhaltung eines Subjektes beiträgt. Kluckhohn (1962, S. 395) definiert Wert als „eine explizite oder implizite, für ein Individuum oder eine Gruppe charakteristische Konzeption des Wünschenswerten, welche die Auswahl unter verfügbaren Handlungsarten, -mitteln und -zielen beeinflusst." Demzufolge sind Werte inhärent subjektiv. Nach Scholl-Schaaf (1975, S. 49-56) ist der Wertbegriff in die folgenden drei Bedeutungsbereiche zu unterteilen, wobei diese nicht als sich auszuschließende Gegensätze zu verstehen, sondern im Zusammenhang zu betrachten sind: (1) substanzielle Werte bzw. Wert als Gut, (2) attributive Werte bzw. Wert als Maßstab, (3) Wert als Ziel. Diese drei Bedeutungsbereiche werden als Grundlage für den weiteren Beitrag charakterisiert.

Substanzielle Werte repräsentieren den Inhalt und die sachliche Festlegung dessen, was angestrebt wird. Attributive Werte sind als Eigenschaft aufzufassen, repräsentieren das Ausmaß der angestrebten Bedürfnisbefriedigung durch eine quantitative oder qualitative Skalengröße und legen das verfolgte Anspruchsniveau fest. Werte sind zunächst abstrakt und von einer konkreten Umsetzung losgelöst. Sie beruhen auf der Basis eines subjektiven Bedürfnisses und motivieren Ziele. Ziele sind zukünftige, als erstrebenswert angesehene Zustände, die durch Handlungen herbeigeführt werden sollen (Macharzina 1999, S. 155). Ziele sind demnach konkrete und bewusste Absichtserklärungen, die auf Werten basieren. Daher können Ziele auch als Repräsentanten von Werten aufgefasst werden. Generell sind die Begriffe Wert, Ziel und Nutzen nur unscharf abgrenzbar, jedoch lassen sich die Begriffe unterschiedlich charakterisieren. Philosophisch betrachtet enthält der Wertbegriff etwas grundlegendes, was um seiner selbst willen angestrebt wird, während Ziele als Mittel zur Realisierung von Werten verstanden werden können. Die Einteilung des Wertbegriffes in substanzielle und attributive Werte finden sich im Zielbegriff in Form von Zielinhalt und Zielausmaß wieder. Der Grad der Zielerreichung wird im Sinne der Höhe des Beitrags der Bedürfnisbefriedigung als eigentlicher Nutzen verstanden. Wertbeitrag ist wie der Zielbegriff an eine Umsetzung gebunden und als tatsächliche Erhöhung des Wertevorrats oder Verminde- 
rung des Werteverlustes aufzufassen. Dies verdeutlicht den engen Zusammenhang der beiden Begriffe. Bezogen auf den Zielbegriff stellt Wertbeitrag ein Wirken zu einem Ziel hin oder der Vermeidung eines Abweichens von einem Ziel dar. Für eine hinreichende Beschreibung des Zielinhaltes ist es notwendig sowohl die sachliche Festlegung des angestrebten Zustandes, als auch die Mittel und Handlungen zu benennen, durch die der Zustand herbeigeführt werden soll. Basierend auf dem vorgestellten abstrakten Wertbegriff wird im folgenden Abschnitt ein betriebswirtschaftlicher Wertbegriff beschrieben.

\subsection{Der betriebswirtschaftliche Wertbegriff}

Der im Rahmen der Betriebswirtschaft verwendete Wertbegriff ist ebenfalls nicht streng definiert. Es existieren unterschiedliche Sichtweisen über dessen Gegenstand. Ein wesentliches Verständnis des Wertbegriffes ist aus dem betrieblichen Leistungserstellungsprozess ableitbar. So definiert Zinsch (2008, S. 5) „Wert“ als den betriebswirtschaftlichen Aspekt des Nutzens, den eine Ressource einem Wirtschaftssubjekt im Leistungserstellungsprozess zuführt. Wert kann daher als InputOutput-Transformationsprozess verstanden werden, dessen Generierung durch die drei Komponenten (1) Ressource, (2) Leistungserstellungsprozess und (3) Nutzen bzw. Ziel beschrieben wird. Diese Wertform wird als Nutzwert bezeichnet (Smart 1923, S. 2; Seppelfricke 2005, S. 1).

Zur Darstellung des Werteverständnisses ist es wichtig, die einzelnen Komponenten strukturiert zu beschreiben. Ressourcen sind Betriebsmittel, die als Inputfaktoren, in den Leistungserstellungsprozess einfließen (Conner 1991, S. 132; Grant 1991, S. 118). Zur Strukturierung des Leistungserstellungsprozesses kann die Wertschöpfungskette nach Porter und Millar (1985) herangezogen werden. Diese umfasst die primären, direkt wertschöpfenden Aktivitäten Eingangs-Logistik, Produktion, Ausgangslogistik, Marketing \& Vertrieb und Kundenservice. Die sekundären Aktivitäten sind indirekt an der Wertschöpfung durch Unterstützung der primären Aktivitäten beteiligt und umfassen die Unternehmensinfrastruktur, Personal, Technologieentwicklung und Beschaffung. Der Nutzen lässt sich entsprechend des im vorherigen Abschnitt dargestellten abstrakten Verständnisses des Wertbegriffes als ein Beitrag zur Zielerreichung im Unternehmen auffassen. Ziele lassen sich in Zielsystemen klassifizieren. Zur Strukturierung schlagen Thommen und Achleitner (2006, S. 113) die in Abb. 1 dargestellte Klassifikation vor, die als Grundlage für eine unternehmensspezifische Anpassung dienen kann.

Neben dem Nutzwert kann Wert noch in einer rein finanziellen Dimension, als monetärer Tauschwert 3 aufgefasst werden. Dieser stellt die Stärke der Fähigkeit dar, sich andere Nutzwerte aneignen zu können. Aufgrund der Transformationsmöglichkeit von Tauschwerten, werden dieser selbst wiederum zu einem nahezu uni-

\footnotetext{
2 Auch als „Value in Use“ oder „Gebrauchswert“ bezeichnet.

${ }^{3}$ Auch als „Value in Exchange“ bezeichnet.
} 
versellen Nutzwert und motiviert Unternehmen zur Zielsetzung der Vermögensmehrung (Zinsch 2008, S. 6).

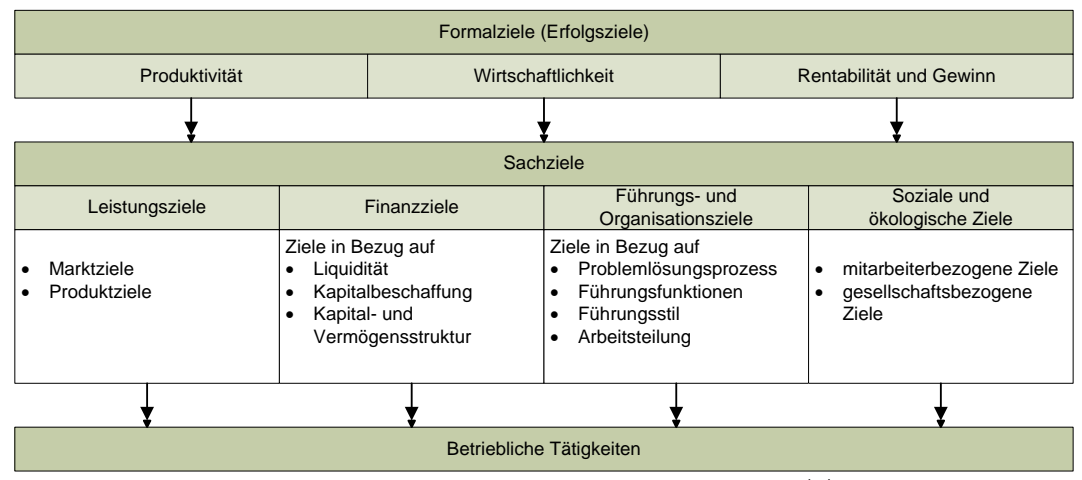

\section{Abbildung 1: Klassifikation von Unternehmenszielen (Thommen und Achleitner 2006, S. 113)}

Traditionell wurde angenommen, dass Gewinn und Liquidität für die langfristige Existenzsicherung von Unternehmen entscheidend sind. Allerdings hat sich die Erkenntnis durchgesetzt, dass auf Buchwerten basierende, Steuerungsgrößen aus verschiedenen Gründen nicht angemessen sind. ${ }^{4}$ Coenenberg und Salfeld (2007, S. 1) stellen fest, dass sich stattdessen das Konzept der Wertorientierung weitestgehend durchgesetzt hat. Dieses stellt das Bestreben der Eigenkapitalgeber (Shareholder) in den Mittelpunkt, den finanziell bewerteten Unternehmenswert und somit den Marktwert ihres eingelegten Kapitals (Shareholder Value) zu maximieren (Thommen und Achleitner 2006, S. 424; Weber 2004, S. 248). In diesem Sinne ist Wertbeitrag als Leistung zu verstehen, die den finanziell bewerteten Unternehmenswert erhöht. Wertorientierung wird als Managementkonzept verstanden, welches sämtliche Aktivitäten auf die langfristige Steigerung des Unternehmenswertes ausrichtet. Eine Ausrichtung aller Aktivitäten (zu denen auch IT Aktivitäten gezählt werden müssen) auf den Shareholder Value ist jedoch als kritisch zu betrachten. Es gibt zwar Ansätze, die das Shareholder Value Konzept in der IT berücksichtigen (vom Brocke et al. 2009), allerdings ist die Möglichkeit der Beurteilung bestimmter Investitionen durch einen monetären Wert nicht oder nur teilweise gegeben (Lucas 1999, S. 203; Baumöl und Ickler 2008, S. 977). Darüber hinaus fokussiert sich das Konzept stark auf die Bedürfnisse der Eigentümer und vernachlässigt weitere Stakeholder ${ }^{5}$. Da der langfristige Fortbestand eines Unternehmens von der Leistung sämtlicher Stakeholder abhängt, ist es sinnvoll, diese in den Wertbegriff mit einzubeziehen. Ulrich und Fluri (1992, S. 77-81) identifizieren Mitarbeiter, Kunden, Manager, Lieferanten, Konkurrenten, Staat/Gesellschaft, sowie Fremdkapitalgeber als weitere Stakeholder. Die Bedürfnisse dieser Stakeholder sind häufig qualitativer Natur und durch Nutzwerte beschreibbar.

\footnotetext{
${ }^{4}$ Für eine genauere Begründung siehe Gleich (2001, S. 6)

${ }_{5}^{5}$ Die Anspruchsgruppen in einem Unternehmen werden als Stakeholder bezeichnet.
} 
Zusammenfassend lässt sich der betriebswirtschaftliche Wertbegriff als Beitrag zur Erreichung sowohl monetärer, als auch qualitativer Unternehmensziele, welche durch sämtliche Stakeholder motiviert sein können, auffassen. Dieser Beitrag wird durch den Einsatz von Ressourcen im betrieblichen Leistungserstellungsprozess generiert. Ausgehend von diesem betriebswirtschaftlichen Werteverständnis, wird der Wertbegriff im Folgenden auf die IT übertragen.

\subsection{Der Wertbegriff und der Wertbeitrag der IT}

Der mit dem Einsatz von Informations- und Kommunikationssystemen zusammenhängende Wertbeitrag wird bereits seit Beginn der Achtziger Jahre diskutiert (Pfeifer 2003, S. 45). Trotz der langjährigen Auseinandersetzung hat sich bisher kein klares Verständnis des Wertbeitrags der IT herausgebildet. Ferner wird auf den Mangel einer adäquaten Definition des Wertbeitrags der IT hingewiesen (Stewart 2003, S. 16). Dieser Mangel und die heterogenen Sichtweisen des Wertbeitrags der IT können auf die erläuterte Subjektivität des Wertbegriffes zurückgeführt werden (Seppelfricke 2005, S. 1; Mirani und Lederer 1998, S. 805-806). Tallon (2000, S. 1) definiert den Wertbeitrag der IT breit als "the contribution of IT to firm performance". Überträgt man den diskutierten betriebswirtschaftlichen Wertbegriff auf die IT, so lässt sich Wertbeitrag als Beitrag zur Erreichung von Unternehmenszielen auffassen, welcher durch den Einsatz von IT Ressourcen im IT Leistungserstellungsprozess erreicht wird. Beide Definitionen sind zwar universell, für ein genaues Verständnis jedoch zu unkonkret. Zur genaueren Beschreibung des Gegenstandes wird der Prozess der Wertgenerierung analog zum betriebswirtschaftlichen Wertbegriff als Input-Output-Transformationsprozess aufgefasst und durch die drei Komponenten (1) IT Ressourcen, (2) IT Organisation und (3) IT Ziel beschrieben. Die IT Organisation bildet den im betriebswirtschaftlichen Wertbegriff erwähnten Leistungserstellungsprozess für die IT ab. Die Komponenten IT Ressourcen und IT Organisation sind als Steuerungsobjekte aufzufassen, welche beeinflussbare Größen darstellen, um die Zielerreichung und den gewünschten Nutzen herbeizuführen.

$\mathrm{Zu}$ (1): Heinrich und Lehner (2005, S. 301) identifizieren Applikationen, ITInfrastruktur und Personal als IT Ressourcen. Aufgrund der zentralen Aufgabe der Informationsversorgung durch die IT, ist die Ressource Information/Wissen als intangibles Betriebsmittel ebenfalls gegenständlich (Barney 1991, S. 101; ITGI 2007, S. 12). Ferner ist anzumerken, dass die Ressourcen in verschiedenen Abstraktionsstufen vorliegen können. Ein Beispiel dafür sind Projektportfolios (Kütz und Friese 2006, S. 23-29).

$\mathrm{Zu}$ (2) Die IT Organisation lässt sich in Aufbau- und Ablauforganisation unterteilen. Ein idealtypische IT Aufbauorganisation besteht aus den Hauptabteilungen Entwicklung, Betrieb und Support (Mertens und Knolmayer 1998, S. 64-68; Kütz und Friese 2006, S. 25). Weitere Hauptabteilungen und Stabstellen wie KeyAccounting, Strategie, Governance sind denkbar und können unternehmensindivi- 
duell berücksichtigt werden. Dies gilt auch für die hierarchisch darunter angesiedelten Abteilungen bis hin zur Stelle als kleinste aufbauorganisatorische Einheit. Die IT Ablauforganisation ist stark unternehmensspezifisch. Es existieren jedoch Frameworks, wie z. B. ITIL und Cobit, welche Gestaltungsvorschläge für die Ablauforganisation beinhalten. Grundsätzlich lässt sich die Ablauforganisation in verschiedene Abstraktionsebenen einteilen. Die kleinste Einheit bildet eine Aktivität, die gebündelt mit einer Abfolge weiterer Aktivitäten einen Prozess bildet. Ein Prozess kann nach Anwendungsdomänen (z. B. Planungsprozesse und MonitoringProzesse) oder kundenorientiert in Services zusammengefasst werden.

$\mathrm{Zu}$ (3): Zur Erläuterung der Komponente der IT Ziele dient das in Abb. 2 dargestellte Modell von Wigand et al. (1997, S. 159). Demnach bilden die Unternehmensstrategie und die daraus ableitbaren Geschäftsprozesse die Basis zur Wertgenerierung. Aus dieser Basis werden Ziele und Anforderungen für die IT abgeleitet (align). Die reziproke Beziehung zwischen IT und Unternehmensstrategie soll verdeutlichen, dass durch die IT ermöglichte Unternehmensstrategien, und Geschäftsprozesse explizit mit berücksichtigt werden (enable). Wesentlich an dem Modell ist jedoch das Verständnis, dass durch den Einsatz von IT keine unmittelbare Wertgenerierung vollzogen wird, sondern IT erst mittelbar, durch die Unterstützung eines Geschäftsprozesses, Wert generiert. Zur Beschreibung in welchem Geschäftsprozess IT Wertbeitrag generiert kann die im vorherigen Abschnitt erläuterte Wertschöpfungskette nach Porter und Millar (1985) herangezogen werden. Da die Geschäftsprozesse selbst wiederum dazu dienen Unternehmensziele umzusetzen, könnte der Wertbeitrag der IT als ein Beitrag zur Zielerreichung eines Geschäftsprozessziels zur mittelbaren Erreichung eines Unternehmensziels aufgefasst werden. Allerdings gibt es Geschäftsprozess-übergreifende Unternehmensziele, die nicht notwendigerweise als Geschäftsprozess-Ziel operationalisiert sind, deren Umsetzung aber durch einen Geschäftsprozess vollzogen wird. Ein Beispiel hierfür wäre ein Wechsel zu einem Open-Source-CRM-System, das eine geringere Kapitalbindung zur Folge hat. Eine reine Fokussierung auf Geschäftsprozessziele als Ausgangsbasis für den Wertbeitrags der IT ist daher nicht hinreichend, so dass neben den Geschäftsprozesszielen auch mittelbare Beiträge zur Umsetzung der Unternehmensziele im Konzept des IT Wertbeitrags berücksichtigt werden müssen. 


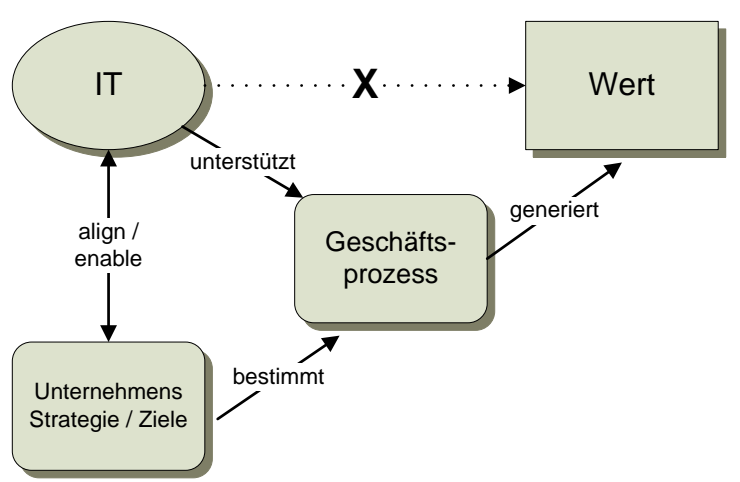

Abbildung 2: Mittelbarer Prozess der Wertgenerierung durch IT (Wigand et al. 1997, S. 159)

Der Wertbeitrag der IT zur Umsetzung von Geschäftsprozess- und Unternehmenszielen kann in die Nutzenkategorien: informationsbezogener, transaktionsbezogener sowie strategischer Beitrag unterteilt werden (Mirani und Lederer 1998, Weill und Aral 2004). Informationsbezogene Beiträge beziehen sich auf eine angemessene Bereitstellung von Informationen im Unternehmen (z. B. ein Business Intelligence System für das Vertriebs-Controlling). Transaktionsbezogene Beitrage werden als Senkung der Transaktionskosten durch Effizienzsteigerungen und Einsparungen verstanden. Strategische Beiträge sind Wettbewerbsvorteile oder Aspekte, welche die Marktmacht des Unternehmens stärken. Das Verständnis des Wertbeitrags der IT als Beitrag zur Zielerreichung der Geschäftsprozess- und Unternehmensziele hat jedoch den Nachteil, dass eine isolierte Verifizierung des erreichten Beitrags durch IT aufgrund weiterer Einflussfaktoren nur bedingt möglich ist (Brown et al. 1995, S. 219). Melville et al. (2004, S. 293) entwickelten auf Basis einer umfangreichen Studie ein deskriptives Modell, welches die Einflussfaktoren der Wertgenerierung auf die Geschäftsprozess- und Unternehmensziele in Unternehmen in einer Ursache-Wirkungskette beschreibt. Sie identifizieren komplementäre organisatorische Einflussfaktoren der Branche, der Geschäftspartner und landesspezifische Charakteristika, die auf Geschäftsprozess- und Unternehmensziele wirken.

Überträgt man den abstrakten Wertbegriff auf die IT, so kann Wertbeitrag auch als Umsetzung der Bedarfe gesehen werden, die sich aus den Geschäftsprozess- und Unternehmenszielen ergeben. Diese Bedarfe werden in Form von Anforderungen an die IT formuliert und stellen aus Sicht der IT interne Ziele dar, ohne den Gegenstand des Geschäftsprozess- oder Unternehmensziels direkt mit einzubeziehen. Anforderungen beschreiben die Beschaffenheit einer Einheit bezüglich ihrer Eignung, festgelegte und abgeleitete Erfordernisse zu erfüllen (Liggesmeyer 2002, S. 5). Zur Spezifizierung der Anforderungen kann z. B. die EN ISO 9000 Normenreihe herangezogen werden. Cobit 4.1 schlägt die Anforderungen Effektivität, Effizienz, Vertraulichkeit, Integrität, Verfügbarkeit, Compliance 
und Verlässlichkeit vor (ITGI 2007, S. 10-11). Zur Beschreibung des Wertbeitrags der IT werden die vorgestellten Komponenten IT Ressourcen, IT Organisation und Ziele samt der dazugehörigen Dimensionen in einem Framework integriert. Die dargestellten Zusammenhänge sind zusammenfassend als Framework zur Klassifikation des Wertbeitrags der IT in Notation der Unified Modeling Language (UML) in Abb. 3 dargestellt. Die Aspekte, die ausschließlich die interne IT Organisation betreffen (IT Steuerungsobjekte und interne IT Ziele) sind weiß dargestellt. Aspekte, die sich ausschließlich auf den Geschäftsbereich beziehen (Geschäftsprozess-Ziel und Unternehmensziel) sind grau dargestellt. Aspekte, die beide Bereiche betreffen können, sind weiß/grau dargestellt.

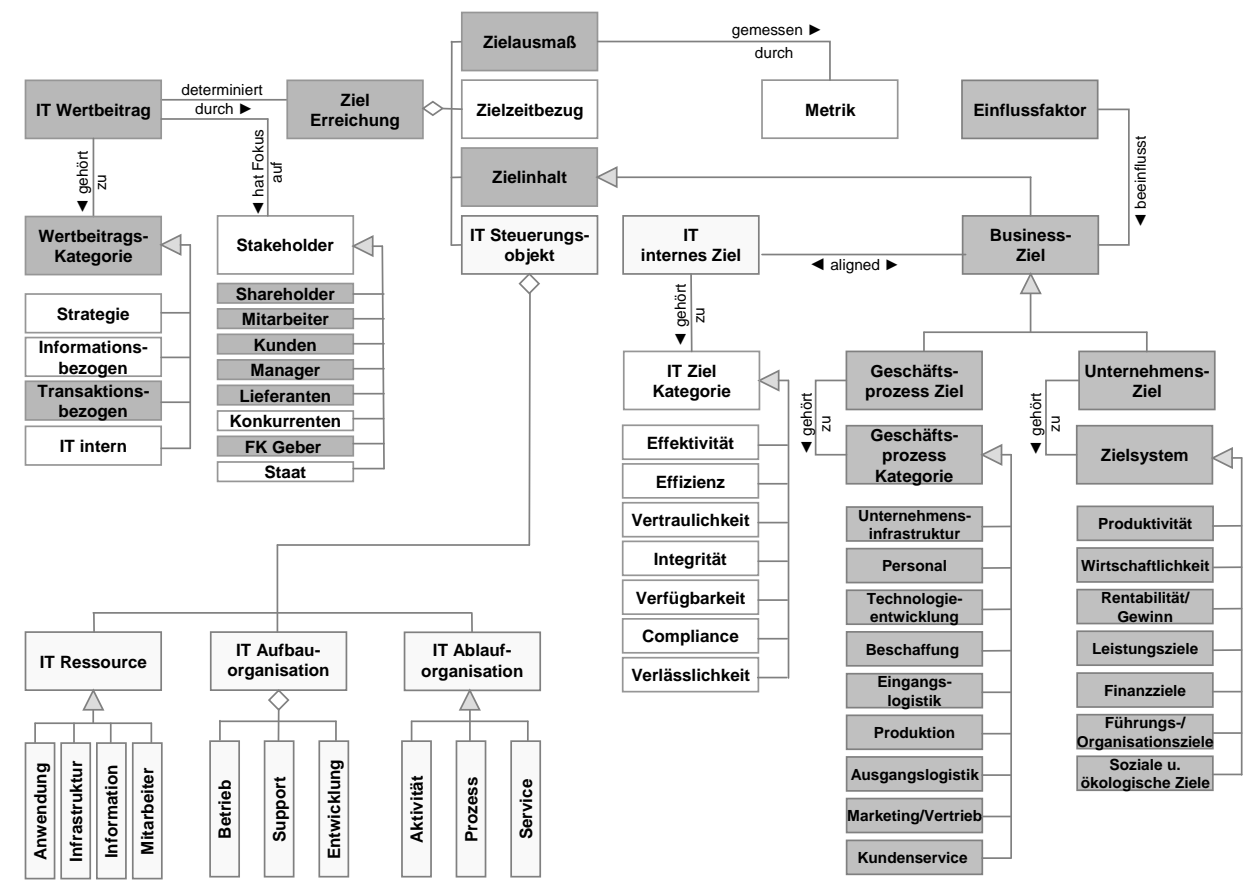

Abbildung 3: Framework zur Klassifikation des Wertbeitrags der IT

\section{Demonstration und Bewertung}

Zur Demonstration wird eine Sichtweise des Wertbeitrags der IT beispielhaft herangezogen und deren Abdeckung durch eine Instanziierung des Frameworks veranschaulicht. Als Beispiel dient eine angestrebte Neuentwicklung eines Webservices für einen Online-Versandhandel. Durch automatische Nachbestellungen bei Zulieferern sollen die Lagerbestände reduziert und dadurch die Kapitalbindung gesenkt werden. Die Kapitalbindung ist ein Hebel zur Erhöhung des Shareholder Values (Coenenberg und Salfeld 2007, S. 169). Der angestrebte Wertbeitrag der IT 
soll aus Sicht der Unternehmensführung und der IT betrachtet werden. Das Beispiel ist in Abb. 4 dargestellt.

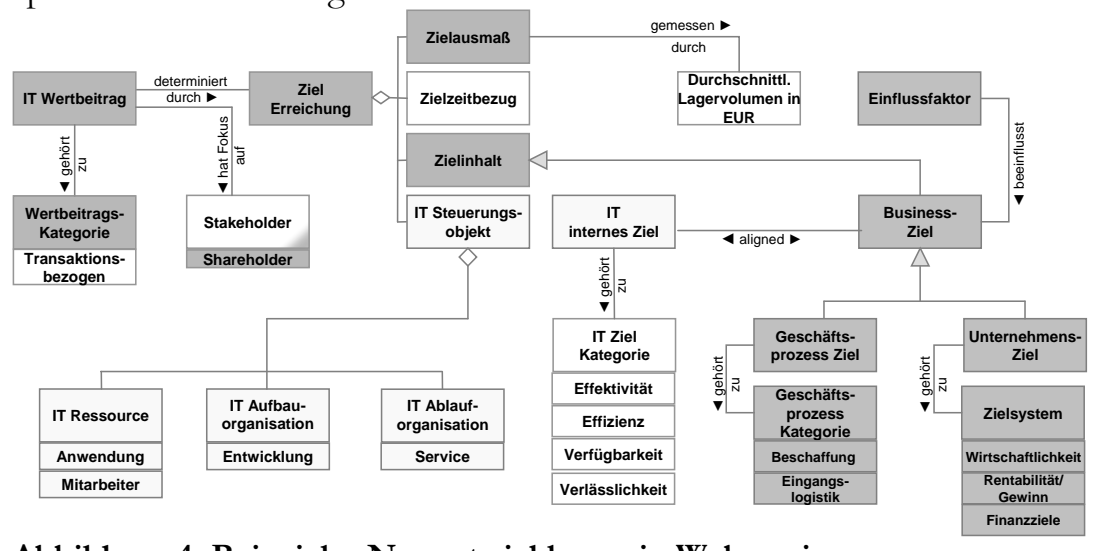

\section{Abbildung 4: Beispiel - Neuentwicklung ein Webservices}

Abschließend lassen sich folgende Punkte festhalten: In diesem Beitrag wurde der Begriff des Wertbeitrags der IT ausgehend von einem abstrakten und einem betriebswirtschaftlichen Wertbegriff hergeleitet und Gründe für die verschiedenen Sichtweisen des Wertebeitrags der IT aufgezeigt. Dabei wurden unterschiedlichen Dimensionen des Wertbeitrags der IT identifiziert und diese in einem Framework integriert. Das Framework bietet einen Ansatz, um die verschiedenen Sichtweisen des Wertbeitrags der IT zu modellieren. Dabei sollen unternehmensspezifische Strukturen (z. B. abweichende Steuerungsobjekte) bewusst in das Framework integriert werden können. Die Demonstration veranschaulicht, dass ein konkretes Problem in einem Kontext gelöst wird. Sie dient einer Veranschaulichung der Anwendung des Modells, klärt im Hinblick auf den zulässigen Umfang jedoch nicht hinreichend in wie weit das Modell tatsächlich geeignet ist, den Gegenstand des Wertbeitrags der IT vollständig abzudecken. Aufgrund der Diversifikation des Gegenstandes ist es auch fraglich, ob dies vollständig gelingen kann. Dennoch bietet das Modell eine Ausgangsbasis, um einem unternehmensspezifischen Wertbeitrag darstellen zu können. Es wäre wünschenswert eine umfangreichere Evaluation durchzuführen, um die Abdeckung weiterer Sichtweisen des Wertbeitrages der IT durch das Framework zu überprüfen und dieses ggf. zu modifizieren. Darüber ist anzumerken, dass alternative Konzeptualisierungen denkbar sind, die den Gegenstand ebenfalls angemessen darstellen.

\section{Literatur}

Bannister F, Remenyi D (2000) Acts of faith: Instinct, value and IT investment decisions. J. Inf. Technol. 15(3):231-241. 
Barney J (1991) Firm resources and sustained competitive advantage. J. Manage. 17(1):99-120.

Baumöl U, Ickler H (2008) Wertorientiertes IT-Controlling Projektselektion als Werkzeug der Projektportfolio-Steuerung. In: Bichler M, Hess T, Krcmar H, Lechner U, Matthes F, Picot A, Speitkamp B, Wolf P (Hrsg) Multikonferenz Wirtschaftsinformatik 2008. Gito, Berlin.

Brown RM, Gatian AW, Hicks Jr JO (1995) Strategic information systems and financial performance. JMIS 11(4):215-248.

Coenenberg AG, Salfeld R (2007) Wertorientierte Unternehmensführung SchäfferPoeschel, Stuttgart.

Conner KR (1991) A historical comparison of resource-based theory and five schools of thought within industrial organization economics: Do we have a new theory of the firm? J. Manage. 17(1):121-154.

Cronk MC, Fitzgerald EP (1997) A conceptual framework for furthering understanding of 'IT business value' and its dimensions. In: Proceedings of 3 rd Pacific Asia conference on information systems, Brisbane.

Gammelgård M, Ekstedt M, Gustafsson P (2006) A categorization of benefits from IS/IT investments http://www.ee.kth.se/php/modules/publications/ reports/2006/IR-EE-ICS_2006_026.pdf. Abruf am 2008-11-24.

Gleich R (2001) Das System des Performance Measurement Vahlen, München.

Grant RM (1991) The resource-based theory of competitive advantage:

Implications for strategy formulation. Calif. Manage. Rev. 33(3):114-135.

Heinrich LJ, Lehner F (2005) Informationsmanagement Oldenbourg, München.

Hevner AR, March ST, Jinsoo P, Ram S (2004) Design science in information systems research. MIS Quarterly 28(1):75-105.

Heyde JE (1926) Wert: eine philosophische Grundlegung Stenger, Erfurt.

ITGI (2007) Cobit 4.1 ISACA, Rolling Meadows.

Johannsen W, Goeken M, Just D, Tami F (2007) Referenzmodelle für ITGovernance Dpunkt, Heidelberg.

Kessinger K, Vohasek D, Duffer J (2009) Nine-country ISACA survey: Two-thirds of companies not fully measuring IT value, neglecting competitive advantage http://www.isaca.org/Template.cfm?Section=Press_Releases1\& CONTENTID $=51580 \&$ TEMPLATE $=/$ ContentManagement $/$ ContentDisplay.cfm. Abruf am 2009-08-10. 
Kleinschmidt P, Pfeifer A (2004) Zur Wirkung von IT/IM in Unternehmen: Analyserahmen, Bestandsaufnahme und Empfehlungen für zukünftige Studien Wirtschaftswissenschaftliche Fakultät, Universität Passau.

Kluckhohn C (1962) Values and value-orientation in the theory of action. In: Parson T, Shils E (Hrsg) Toward a General Theory of Action. Harvard University Press, Cambridge.

Krcmar H (2005) Informationsmanagement Springer, Berlin.

Kütz M, Friese P (2006) IT-Steuerung mit Kennzahlensystemen Dpunkt, Heidelberg.

Liggesmeyer P (2002) Software-Qualität Spektrum, Akad. Verl, Heidelberg.

Lucas HC (1999) Information technology and the productivity paradox Oxford Univ. Press, New York.

Macharzina K (1999) Unternehmensführung Gabler, Wiesbaden.

Melville N, Kraemer K, Gurbaxani V (2004) Review: Information technology and organizational performance: An integrative model of IT business value. MIS Quarterly 28(2):283-322.

Mertens P, Knolmayer GF (1998) Organisation der Informationsverarbeitung Gabler, Wiesbaden.

Mirani R, Lederer AL (1998) An instrument for assessing the organizational benefits of IS projects. Decision Sciences 29(4):803-838.

Peffers K, Tuunanen T, Rothenberger MA, Chatterjee S (2007) A design science research methodology for information systems research. JMIS 24(3):45-77.

Pfeifer A (2003) Zum Wertbeitrag von Informationstechnologie. http://www.opus-bayern.de/uni-passau/volltexte/2004/34/pdf/ PfeiferAndreas.PDF. Abruf am 2006-05-08.

Porter ME, Millar VE (1985) How information gives you competitive advantage. Harvard Bus. Rev. 63(4):149-174.

Scholl-Schaaf M (1975) Werthaltung und Wertsystem Bouvier, Bonn.

Seppelfricke P (2005) Handbuch Aktien- und Unternehmensbewertung SchäfferPoeschel, Stuttgart.

Smart W (1923) An introduction to the theory of value on the lines of Menger, Wieser, and Böhm-Bawerk Macmillan, London.

Stewart RA (2003) Lifecycle management of information technology (IT) projects in construction. http://www4.gu.edu.au:8080/adt-root/uploads/approved/ adt-QGU20030423.122317/public/04Chapter5-6.pdf. Abruf am 2009-01-29. 
Tallon PP, Kraemer KL, Gurbaxani V (2000) Executives perceptions of the business value of information technology: A process-oriented approach. JMIS 16(4):145-173.

Thommen J-P, Achleitner A-K (2006) Allgemeine Betriebswirtschaftslehre Gabler, Wiesbaden.

Ulrich P, Fluri E (1992) Management Haupt, Bern.

vom Brocke J, Sonnenberg C, Simons A (2009) Wertorientierte Gestaltung von Informationssystemen: Konzeption und Anwendung einer

Potenzialmodellierung am Beispiel Serviceorientierter Architekturen WIRTSCHAFTSINFORMATIK 51(3):261-272.

Weber J (2004) Einführung in das Controlling Schäffer-Poeschel, Stuttgart.

Weill P, Aral S (2004) Managing the IT portfolio: Returns from the different IT asset classes. MIT CISR IV(1):1-4.

Wigand R, Picot A, Reichwald R (1997) Information, organization and management Wiley, Chichester.

Zinsch BA (2008) Bewertung mittelständischer Unternehmen Diplomica-Verl, Hamburg. 